suggested a higher prevalence of Body Dysmorphic Disorder in patients with Acne Vulgaris which should be considered and screened for.

Conclusion. This scoping literature review has highlighted the significant psychological burdensome acne patients can experience. Given the prevalence of the condition Psychiatrists do have a role in working with Dermatologists to ensure appropriate screening tools are utilised and patients are able to access appropriate support.

\section{Factors associated with mental health outcomes among medical residents exposed to COVID-19}

Mohamed Adil Shah Khoodoruth ${ }^{1 \star}$, Sami Ouanes ${ }^{1}$, Malek Smida ${ }^{1}$, Zerak Al-Salihy ${ }^{1}$, Saleem Al Nuaimi ${ }^{1}$, Widaad Nuzhah Chut-kai Khoodoruth ${ }^{2}$, Adeel Ghaffar ${ }^{2}$ and Mohammed Faisal Hamad Mohammed ${ }^{1}$

${ }^{1}$ Psychiatry Hospital, Hamad Medical Corporation and

${ }^{2}$ Hamad Medical Corporation

${ }^{*}$ Corresponding author.

doi: 10.1192/bjo.2021.617

Aims. The aims of our study were to assess and to examine i. the psychological impact of the COVID-19 pandemic on medical residents working on the front and second line, ii. the association between coping strategies, resilience and optimism and different mental health outcomes like stress, anxiety, and depression among the medical residents' workers during the COVID-19 pandemic, and iii. the coping strategies used on the same sample with consideration of different factors like seniority, frontliner, gender and coping style.

Method. An electronic survey was sent to all medical residents in Qatar. Depression, anxiety and stress were assessed by the Depression, Anxiety and Stress Scale - 21 Items. Professional quality of life was measured by the Professional Quality of Life measure. The coping mechanisms were assessed with the Brief-COPE, resilience by the Brief Resilience Scale, and optimism by the Revised Life Orientation Test (LOT-R).

Result. Of the 640 medical residents contacted, 127 (20\%) responded. A considerable proportion of residents reported symptoms of depression (42.5\%), anxiety (41.7\%) and stress (30.7\%). Multivariate analysis of variance showed significant effects of seniority in residency, with junior residents having poorer outcomes. In addition, there was a statistically significant interaction effect with moderate effect sizes between gender and working on the front line, as well as gender, working on the front line and seniority, on mental health outcomes. The most commonly used coping strategies were acceptance, religion, and active coping. The least reported coping strategies were substance use and denial. Avoidant coping style scores were higher among junior residents $(\mathrm{p}=.032)$ and non-COVID-19 frontliners $(\mathrm{p}=.039)$. Optimism LOT-R score was higher in senior than in junior residents $(\mathrm{p}<.001)$. Another important finding is that optimism and resilience were associated with better mental health outcomes. In addition, we find that avoidant coping style is highly associated with depression.

Conclusion. The COVID-19 pandemic may have a negative impact on junior residents' mental health. Preventive measures to reduce stress levels and easy access to professional mental health services are crucial. This study also raises awareness among residency programs on the psychological and coping responses and strategies of medical residents.
GP Trainees' perceptions and experiences of the training placement in Psychiatry - A qualitative study

Raja Adnan Ahmed ${ }^{1 \star}$, Rugiyya Saeed ${ }^{2}$ and Michal Tombs ${ }^{3}$

${ }^{1}$ Aneurin Bevan University Health Board; ${ }^{2}$ University Hospital of Wales and ${ }^{3}$ Cardiff University

${ }^{\star}$ Corresponding author.

doi: 10.1192/bjo.2021.618

Aims. This qualitative study aims to explore the leaning needs of the GP trainees for their psychiatry placements.

Method. In this qualitative study, semi-structured interviews of eight former GP trainees were conducted. Data were transcribed and analysed using thematic analysis. Triangulation through multiple analysts" was used to improve the validity of the study

Result. This study identified six key areas of learning needs for GP trainees during their psychiatry placement. i) the on-call experience which enabled the participants to learn how to manage acutely unwell patients in psychiatry, ii) learning the self-harm and suicidal risk assessment which is an important skill for a GP practising in primary care, iii) training in relation to psychiatric medication which enabled GPs to prescribe more confidently in the community, iv) exposure to the community psychiatry which was helpful in getting exposure to community-based clinical practice, v) learning from formal teaching activities which can be tailored to cover the relevant primary care related clinical topics and finally, vi) getting the opportunity to improve the communications skills during the psychiatry placement which is useful for all doctor in training.

Conclusion. We recommend that detailed induction of the service setup is required before GP trainees start on-calls and a well-defined support network should be provided and explained to the training doctors. Risk assessment teaching should be delivered by formal training, regular supervision and discussions. Training on psychiatric medication especially in the context of GP prescribing should be considered as part of formal teaching experience. Opportunities to work with community mental health teams and outpatient clinics should be generated and offered to the trainees. Formal teaching sessions should be set up with an understanding of the GP training curriculum and their learning needs. Improvement of communications skills with exposure to difficult communication scenarios under supervision during psychiatric placement should be identified as an important area of learning for the GP trainees.

\section{Psychological impact of COVID-19 pandemic on frontline health care workers in Bangladesh: A cross-sectional study}

Suman Ahmed ${ }^{1 \star}$, Mohammad Shamsul Ahsan², Rubaiya Khan ${ }^{2}$, Mahbubul Hasan ${ }^{2}$, Fahmida Ferdous², Humayra Shahjahan ${ }^{2}$, Murin Hossain ${ }^{2}$, Ananya $\mathrm{Kar}^{2}$ and Kamrul Hossain ${ }^{3}$

${ }^{1}$ Tees, Esk and Wear Valleys NHS Foundation Trust; ${ }^{2}$ Bangabandhu Sheikh Mujib Medical University and ${ }^{3}$ Daffodil International University

${ }^{*}$ Corresponding author.

doi: 10.1192/bjo.2021.621

Aims. Frontline health care workers exposed to COVID-19 patients could be at increased risk of developing psychological issues. The study aimed to estimate the prevalence of mental health-related problems, specifically depression, anxiety, post-traumatic stress disorder (PTSD), and insomnia among health care professionals during the COVID-19 pandemic in Bangladesh and to compare these between medical and allied health care professionals. 